\title{
Recommended guidelines for studies of human subjects with spinal cord injury
}

Spinal Cord (2005) 43, 453-458. doi:10.1038/sj.sc.3101746; published online 12 April 2005

Note: This document has taken into consideration guidelines previously recommended by the American Society for Neural Transplantation and Repair ${ }^{1}$ for translational studies in the nervous system. Some contents of the previous document have been reproduced verbatim in this document, with permission.

Goals: Research to discover therapies for spinal cord injury (SCI) has made steady progress over the last several years. Preclinical reports of neuroprotection, regeneration and functional recovery have prompted several investigators to begin planning for anticipated clinical trials, whereas other groups have already initiated such trials. The purpose of this position paper is to outline issues that should be considered in the planning, initiation and conduct of human clinical trials in SCI.

\section{General considerations}

Clinical trial plans should conform to established and generally accepted standards for the conduct of clinical trials, and must meet the regulatory requirements of the host country in which the trials are conducted. Investigators performing clinical trials could benefit from learning about and meeting regulatory requirements of other countries as well. Every study should meet requirements for the enrollment of human subjects in research as outlined by the Declaration of Helsinki and subsequent Belmont Report. In addition, it is recommended that clinical trials conform to the recommendations outlined below.

\section{Preclinical studies}

Appropriate data should be acquired in animals prior to human studies. In general, the proposed clinical trial should be based on supportive preclinical animal efficacy data of a nature that would be considered predictive of lasting clinical benefit. The nature of the recommended preclinical model may depend on the timing of intervention (acute, subacute, or chronic intervention), and the risk/benefit ratio of the proposed intervention.

Spinal cord damage can result from many causes, including acute traumatic compression/contusion, penetrating trauma, transient or prolonged compression, ischemia, anoxia, infection, immune and inflammatory responses. Contusive trauma is the most common form of acute spinal cord injury and is best modeled by spinal cord compression/contusion models that result in cavitation. If a clinical trial is to be performed in patients with contusive-type injuries, preclinical validation should also be established in animal contusion injury models. Human trials of treatments for other causes of spinal cord damage should ideally be tested in animal models representative of these other forms of damage (eg, anoxia).

A proposed acute/subacute intervention should be tested in an injury model replicating the time frame of the proposed treatment. For example, if treatment is to be rendered within $24 \mathrm{~h}$ in an experimental neuroprotective strategy, the animal model should test time points that include delays of up to $24 \mathrm{~h}$ postinjury. If a repair therapy is designed for delivery within two weeks of injury, the animal model should be studied at time points that include delays of treatment up to two weeks.

A proposed chronic regenerative therapy should be tested in a chronic injury model. Most known degenerative events and scar-related events caused by acute injury under laboratory conditions in rodents have stabilized by 3 months postinjury. Thus, unless the time window for the targeted event is known to be different, 3-month or longer time periods constitute optimal rodent data for a chronic study. A number of degenerative events have stabilized by 6 weeks postinjury, and this would represent a feasible but less desirable time point in which to test a chronic intervention in rodent models. However long the post-injury period, inclusion of appropriate matched control groups should still be considered an essential requirement.

Proposed interventions that are highly invasive or of greater intrinsic risk must meet a higher standard of preclinical safety and efficacy than interventions of lower risk. For example, surgical manipulation at or above a cervical lesion places critical functional neural substrates at risk. On the other hand, oral therapy with currently approved pharmacological agents having an established profile of excellent safety constitutes a lower risk and can undergo novel clinical trials after meeting simpler requirements. More invasive or higher risk protocols in particular should be tested in larger animal models than rodents, including feline, canine, porcine or primate models of injury. Interventions to be applied to cervical levels of injury in humans should be tested at cervical levels of injury in animals. 
When an intervention involves cell grafting, consideration should be given to potential differences that may result from paradigms employing autografts, allografts or xenografts. Immune considerations may influence graft survival, inflammation and efficacy.

Any proposed treatment should be of clearly demonstrable and statistically significant benefit in animal models, and should be peer-reviewed by independent experts. It is preferable that the preclinical findings be published in peer-reviewed journals and should be independently replicated by individuals experienced in the field. Safety issues must be addressed, as outlined below.

Clinical trials should never be conducted when validation from animal models is entirely lacking.

Safety issues for consideration related to novel treatments (1) Any treatment: (For brevity, we refer to proposed or putative therapies as 'therapies' in the guidelines that follow.) Safety assessments should be documented and should optimally include outcome measures testing pain, as well as assessing maintenance of function in systems above the lesion. The period of observation post-treatment should be sufficient to document freedom from significant adverse events, and would preferably extend for at least 6 months to 1 year. Therapies applied to humans with partial lesions risk loss of spared function, and interventions intended for application to these human subjects should include safety assessments in preclinical animal models of partial injury. Treatments delivered to the cervical cord risk compromising critical, spared neurological function, and novel treatments should only be applied to this level of the cord if it is certain that they do not exacerbate damage to the cord.

(2) Cell-based therapies: Any cell source, including olfactory ensheathing cells, stem cells, Schwann cells, white blood cells, bone marrow stromal cells, fibroblasts, astrocytes, fetal tissue or other tissue should be tested for possible biological (cellular, bacterial, viral, prion) contamination before placement in humans. For cell types with the potential for uncontrolled growth, highly defined and restricted cell sources should be used and tested in animal models to evaluate the risk of tumor formation or undesirable cell migration. Ideally, safe methods to eliminate implanted cells in the event of a serious adverse effect would be integrated into the delivery method, as the technology for doing so is developed. Additional tests for genetic instability are desirable, include karyotypic banding and sky analysis. Cellular products with the potential for differentiation should be tested and characterized for these possibilities in the neural location intended for use. Again, the duration of post-treatment observation should be sufficient to reasonably predict freedom from adverse events, preferably at least 6 months.

(3) Pharmacological therapies: Systemic side effects of pharmacological therapies should be assessed, including cardiovascular, pulmonary, renal, hematopoietic, endo- crine and gastrointestinal function. In addition, agents applied either systemically, intrathecally or intraparenchymally should be assessed for risk of eliciting immune responses (if foreign to mammalian systems, eg, chondroitinase) and for safety as outlined in point 1 above.

(4) Therapies aiming to neutralize inhibitors: The normal function of inhibitors to axon growth that are associated with myelin, or that are present in the extracellular matrix, of the intact adult nervous system is unknown. It is possible that neutralization of these inhibitors might unleash unintended growth or dysfunctional plasticity, resulting in aberrant function in neural circuitry. For this reason, therapies aimed at neutralizing these inhibitors should be screened for both the potential to cause toxicity (seizures, disruption of cognitive function, pain) and, in the case of myelindirected therapies, for the risk of demyelination. Optimally, these neutralization therapies should be targeted specifically to the spinal injury site and should not influence other CNS regions.

(5) Growth factors: Growth factors normally support the function of neuronal systems. It is possible that the therapeutic delivery of growth factors could perturb the function of normal neurons or affect sensory pathways, including pain. Optimally, growth factor therapy should be targeted specifically to those regions affected by injury.

(6) Gene therapy: There should be justification and supporting preclinical data for the proposed choice of delivery system or vector, compared with other possibilities. Toxicity of vector constructs and any gene products on cells, tissue and animals should be identified and balanced against the possible benefits. The absence of vector effects on germ cells and progeny should be demonstrated, as well as the absence of vector/viral replication or reversion to wild-type virus. The spread of vector in the host that depends on host mechanisms, such as retrograde transport, should be determined specifically in the most appropriate animal species, and possible adverse consequences considered. The spatial, temporal and quantitative analyses of transgene expression should be determined and should be adequate to expect a useful therapeutic effect. Further, the consequences of extended, unregulated and supraphysiological transgene expression from vectors should be considered and justified if there is no means for regulating transgene expression or activity. Safety assessment for gene-based therapies may require longerterm follow-up than is typical in animal experiments.

(7) Ex vivo gene therapy: For the combination of gene and cell-based interventions, considerations outlined in points 1,2 and 6 should all be addressed.

\section{Evidence of beneficial effects}

Ideally, outcomes should be assessed at cellular, physiological and behavioral levels in a model of SCI that is most appropriate for the indication. At a minimum, evidence of functional benefit should be 
demonstrated on accepted, objective measures of functional outcome. Functional improvement in the animal model should be of sufficient magnitude and duration to justify the potential risk of a clinical trial.

Persistence of a therapeutic benefit should be shown for at least 3 months post-injury to ensure that there are true differences between treated and untreated animals, in order to justify the inherent risks of proceeding with a clinical trial.

As noted above, publication of findings after peerreview is highly desirable prior to initiation of clinical trials. Optimally, evidence of independent replication should be presented prior to initiation of clinical trials. This requires not only complete disclosure of methodological details in publications, but ideally cooperation between the initiating laboratory and those undertaking the replication.

Significant systemic effects or other undesirable effects should be screened for carefully and reported in a public format. It should be appreciated that unexpected adverse effects are frequently encountered in human clinical trials, and animal models should be carefully monitored for adverse effects. For example, pain, worsened autonomic dysfunction or spasticity could result from enhanced axonal plasticity after injury and should be screened for prior to initiation of clinical trials, as noted above.

The clinical benefit of methylprednisolone in SCI remains a subject of debate. Whether a potential therapy must be compared to a group of parallel subjects treated with methylprednisolone remains unresolved. This matter will likely be determined on a case-by-case basis in individual trials depending on the nature of an experimental therapy, local standards, and in consultation with regulatory agencies.

\section{Clinical studies}

The design and conduct of the study should be in full compliance with local and national standards. For example, in the United States, clinical trials should meet requirements for submission and approval by the US Food and Drug Administration, Institutional Review Boards (Human Subjects Research Committees), and Biosafety Committees. Every trial, internationally, must meet requirements for enrollment of human subjects in medical research as outlined by the Declaration of Helsinki and subsequent Belmont Report, including absolute requirements for informed consent. The following study design issues should be considered.

\section{Study phases; importance of blinded assessment}

Studies should follow the model of sequential: (1) safety assessment (Phase I), (2) early measurement of treatment activity and dose/regimen selection (Phase II) and (3) definitive assessment of safety and efficacy (Phase III).

Extraordinary care should be taken, particularly in phase II and III trials, to employ objective functional outcomes measures and appropriate placebo and blinded assessments by trained assessors to avoid possible participant or experimenter bias. When doubleblind designs are not feasible, for example, a test of behavioral training paradigms, inclusion of blinded assessments remains preferable. External study monitoring should be performed.

\section{Study duration}

Improvement should be judged to be significant at a time point that is conclusive.

In subjects with acute SCI treatments, efficacy outcomes should be assessed optimally at the 1-year postinjury time point. In subjects with stable chronic injuries, outcomes should be assessed optimally at 1year post-intervention, although 3- and 6-month postinjury time points also can be informative.

\section{Study size}

Studies must be large enough and consist of sufficient control subjects in phases II and III trials to yield meaningful data. Group size will depend on the magnitude of the potential effect of the manipulation. In general, conclusions regarding efficacy should not be generated from small number of patients, particularly in phase I safety trials.

\section{The confounding effect of rehabilitation training and placebo}

Outcomes after experimental SCI trials in humans can potentially be affected by variables other than true efficacy of the tested therapy, including placebo effects, prior medical care, surgical methods, rehabilitation training and spontaneous improvement over time. It is critical that SCI studies rigorously control these variables.

Whenever possible, experimental trials should use placebo control groups. In the case of invasive treatments, placebos can consist of simple skin incisions. Double blinding is important and should be included in the design of efficacy trials whenever feasible. Every attempt should be made to extend blinding of treatment group identity to subjects, therapists, assessors and as many other study personnel as possible.

Outcomes can be affected by methods and procedures used intraoperatively that are distinct from the actual experimental treatment, including decompression of the lesion, cord detethering, stabilization, or the 'routine' use of substances such as gelfoam, which contain bioactive materials. Ideally, these variables would be controlled between trials by consensus reached among trialists. At the least, surgical methods should be stated in complete detail when study results are reported.

It is believed that intensity of physical rehabilitation for specific disabilities can affect such parameters as spasticity, the function of systems spared by injury, or the number of medical complications, and thus, affect 
outcomes of experimental trials. It is critical, therefore, that clinical trials match experimental and control groups for physical therapy and other physical activities, and consider the possibility that physical therapy may synergize with other therapeutic manipulations to affect clinical trial outcomes. To the extent that is practical, other features of postoperative care should be discussed among clinical trialists and matched between trials, including choice of antibiotics, use of traction and time to mobilization. In addition, to the extent possible, controls should be matched for age and gender.

Spontaneous or natural improvement in function can occur after SCI. It is therefore important to rigorously compare outcomes to appropriate control groups assessed at similar time points of at least one year postinjury. See the section 'study duration'.

\section{Comparison of experimental therapies to} methylprednisolone or future, effective therapies

As noted above, the benefit of treatment of acute SCI with methylprednisolone remains a subject of debate. At present, it may not be necessary to include a clinical group treated with methylprednisolone in clinical trials of novel therapies.

It is possible that new therapies will emerge that demonstrate efficacy for the treatment of SCI. In such a case, subsequent clinical trials should compare outcomes of patients treated with the new experimental therapy to any previously identified, effective therapy.

\section{Subject selection, assignment to study group, statistical analysis}

The criteria for participant selection should be defined so as to include the appropriate population for assessment of clinical benefit. For example, a therapy that aims to enhance plasticity of spared systems below an injury should be tested in groups predivided into 'clinically incomplete' and 'complete' subjects, to enhance the validity of statistical analysis. Experiments in chronically injured subjects may be most safely tested in subjects with clinically complete lesions, and thoracic injury levels, before proceeding to enroll subjects with clinically incomplete lesions or cervical injuries.

Consideration should be given to excluding subjects that might confound interpretation or that might suffer excessive adverse events during the study.

Subjects should be randomly assigned to active or control groups; including all randomized subjects in intent-to-treat analyses is a preferred design.

Plans for data collection and statistical analyses should be included in the proposed study design, and data management resources should be adequate for all aspects of the studies.

If trials are conducted in global regions lacking resources to meet these criteria, consideration should be given to recruiting study collaborators with the background, experience and resources to meet the preceding standards for clinical trial conduct. Clinical trials that are conducted without meeting these criteria present the risk of confounding rather than promoting progress toward the goal of identifying effective therapies for SCI.

\section{Control groups}

Control groups should be included in the clinical trial design whenever possible, as noted above; however, control groups that would be ideal for animal studies might be inappropriate for human studies. Possible experimental controls might include sham surgery extending up to, but not beyond, the bounds of reasonable risk. For example, a bed-bound SCI patient who has been recently injured may tolerate no more than a simple skin incision, yet this procedure would accomplish the objective of double blinding with minimal risk. A paraplegic patient in later stages of injury and good health may tolerate, with minimal risk, a longer period of anesthesia or sedation with a larger skin incision. Subjects in a control group could be offered the experimental treatment once the blinded portion of a study is completed.

These considerations should be guided by the principle that harm is minimized to study participants whenever possible, consistent with achieving an unbiased assessment of outcome. A small risk is generally considered acceptable in clinical trials to obtain an unbiased assessment of outcome.

\section{Immune problems and rejection}

Several experimental treatment strategies carry the risk of producing an immune response in the recipient. The risk-benefits of using immunosuppressive drugs should be assessed; whether temporary immunosuppression is feasible should be explored in preclinical studies. The research plan should consider how an immune response would be measured and prevented, how it would impact study outcome, and how immune response in the recipient will affect future well-being.

\section{Standardized assessment methods should be used}

Studies should use assessment methods comparable to those of other investigators. The nature of optimal outcome measures for clinical trials in SCI remains a subject of ongoing discussion and revision. At present, the ASIA grade (A-E) and motor and sensory scales for inclusion/exclusion, stratification and general outcomes are considered standard; they should be included in clinical trial designs to create baseline information that can be compared across trials and any historical data that may exist. Outcome measures should be relevant and sensitive to the impairments and disabilities that may change in relationship to differing levels of cervical or thoracolumbar SCI. Pain threshold, allodynia, spasticity (the Ashworth scale) and frequency of autonomic dysreflexia should be monitored. Measures of functional independence, quality of life and bowel/ 
bladder function are desirable. Ventilatory function relating to both inspiratory phrenic function and cough reflexes must be monitored if manipulating the cervical spinal cord.

\section{Outcome measures other than clinical physical examination}

Markers of disease extent or treatment efficacy, other than the neurological examination, can be incorporated into clinical trial design. These can include new functional scales, electrophysiological assessments (eg, sensory-evoked potentials, magnetic-evoked stimulation) and structural and/or functional MR imaging. However, there should be consensus in the field that new outcome measures are valid and generally acceptable.

\section{Informed consent}

Each subject involved in a clinical study should give appropriate consent for participation in the study, being fully informed as to whether the study aims to produce basic scientific knowledge, disease-specific knowledge or potential individual therapeutic benefit to the participant. The possible negative outcomes should be clearly and simply stated. The Informed Consent document must be approved by the Institutional Review Board, and any other required regulatory oversight committee.

In general, patients should be made aware of the fact that the experimental therapy is being tested to determine whether it can 'improve' their condition, but that it is extremely unlikely to constitute a complete 'cure.'

\section{Conflict of interest}

Commercial patentable interests should not operate to the detriment of the free sharing of scientific information and the adherence to the above goals and considerations. It is in the best interest of society and of patients that studies are carried out in the best manner possible, and that results, both positive and negative, are communicated by publication after a reasonably brief period of time.

Financial support for, and potential conflicts of interest by, investigators should be fully disclosed to potential human subjects. These should also be disclosed during presentations at scientific meetings and in publications. Potential conflicts include the ownership of a patent interest, stock or stock options, paid consultant arrangements, or membership on Scientific Advisory Boards of companies which might benefit from the study.

\section{Publication and communication of results}

The optimal mechanism for free and open communication of study results to the scientific community includes presentation at peer-reviewed meetings and peerreviewed publications. Communication of study out- comes to the general public through press releases, news conference, interviews with reporters or presentation to lay groups, prior to publication of results in a peerreviewed journal, deprives the public of the protection of the peer-review process and may jeopardize appropriate publication in leading journals. This process may also mislead patients and reflect unfavorably on the scientists, clinical investigators and the entire field. These consequences are particularly unfortunate for clinical studies in our field and should be avoided.

Public disclosure of (incomplete) interim data can constitute a conflict of interest. Results of studies should generally not be advertised or presented publicly until the study is complete and data fully analyzed. Findings of uncontrolled Phase I studies are extremely difficult to interpret, and claims of potential efficacy are generally unwarranted at this stage.

DK Anderson ${ }^{1}$, M Beattie ${ }^{2}$, A Blesch $^{3}$, J Bresnahan $^{2}$, M Bunge ${ }^{4}$, D Dietrich ${ }^{4}, \mathrm{~V}$ Dietz $^{5}$, B Dobkin ${ }^{6}$, J Fawcett $^{7}$, M Fehlings ${ }^{8}$, I Fischer ${ }^{9}$, R Grossman ${ }^{10}$ J Guest $^{4}$, T Hagg ${ }^{11}$, ED Hall ${ }^{12}$, J Houle ${ }^{13}$, $\mathrm{N}_{\text {Kleitman }}{ }^{14}$, J McDonald ${ }^{15}$, M Murray9', A Privat ${ }^{16}$, P Reier ${ }^{1}$, J Steeves ${ }^{17}$, O Steward ${ }^{18}$, W Tetzlaff ${ }^{17}$, MH Tuszynski*,3, SG Waxman ${ }^{19}$, S Whittemore ${ }^{11}$, J Wolpaw ${ }^{20}$, W Young ${ }^{21}$ and B Zheng ${ }^{22}$ ${ }^{1}$ University of Florida, Gainesville, FL, USA; ${ }^{2}$ Ohio State University, Columbus, $O H, U S A ;{ }^{3}$ University of California-San Diego, La Jolla, CA, USA; ${ }^{4}$ The Miami Project, Miami, FL, USA; ${ }^{5}$ University Hospital Balgrist, Zürich, Switzerland; ${ }^{6}$ University of California-Los Angeles, CA, USA; ${ }^{7}$ Cambridge University, $U K$;

${ }^{8}$ University of Toronto, Canada; ${ }^{9}$ Drexel University, Philadelphia, PA, USA; ${ }^{10}$ Baylor University, Houston, $T X, U S A ;{ }^{11}$ University of Louisville, KY,USA;

${ }^{12}$ University of Kentucky, Lexington, $K Y, U S A$;

${ }^{13}$ University of Arkansas, Little Rock, AR, USA; ${ }^{14}$ The

National Institutes for Health, Bethesda, MA, USA;

${ }^{15}$ Johns Hopkins University, Baltimore, MA, USA;

${ }^{16}$ Montpelier, France; ${ }^{17}$ University of British Columbia, Vancouver, Canada; ${ }^{18}$ University of California-Irvine, CA, USA; ${ }^{19}$ Yale University, New Haven, CT, USA;

${ }^{20}$ Wadsworth Center, New York State Department of Health, Albany, NY, USA; ${ }^{21}$ Rutgers University, Newark, NJ, USA; ${ }^{22}$ Stanford University, CA, USA

*Correspondence: MH Tuszynski, University of California, San Diego, La Jolla, CA 92093-0626, USA

Authors listed alphabetically

\section{References and other useful references and documents}

1 Redmond DE et al. The American Society for Neural Transplantation and Repair considerations and guidelines for studies of human subjects. Cell Transplant 2001; 10: 661-664. 
2 Bracken MB et al. A randomized, controlled trial of methylprednisolone or naloxone in the treatment of acute spinal-cord injury. NY Med J 1990; 322: 1405-1411.

3 Young W, Bracken MB. The Second National Acute Spinal Cord Injury Study. J Neurotrauma 1992; 9 (Suppl 1): S397-S405.

4 Maynard Jr FM et al. International standards for neurological and functional classification of spinal cord injury. American Spinal Injury Association. Spinal Cord 1997; 35: 266-274.

5 Ditunno Jr JF, Graziani V, Tessler A. Neurological assessment in spinal cord injury. Adv Neurol 1997; 72: 325-333.

6 Metz GA et al. Validation of the weight-drop contusion model in rats: a comparative study of human spinal cord injury. J Neurotrauma 2000; 17: 1-17.

7 Ramer MS, Harper GP, Bradbury EJ. Progress in spinal cord research - a refined strategy for the International Spinal Research Trust. Spinal Cord 2000; 38: 449-472.

8 Ditunno Jr JF et al. Walking index for spinal cord injury (WISCI): an international multicenter validity and reliability study. Spinal Cord 2000; 38: 234-243.

9 Geisler FH, Coleman WP, Grieco G, Poonian D. Recruitment and early treatment in a multicenter study of acute spinal cord injury. Spine 2001; 26: S58-S67.

10 Neurosurgery. Pharmacological therapy after acute cervical spinal cord injury. Neurosurgery 2002; 50: S63-S72.
11 Bracken MB. Steroids for acute spinal cord injury. Cochrane Database Syst Rev. CD001046 (2002).

12 Dobkin $\mathrm{BH}$ et al. Methods for a randomized trial of weight-supported treadmill training versus conventional training for walking during inpatient rehabilitation after incomplete traumatic spinal cord injury. Neurorehabil Neural Repair 2003; 17: 153-167.

13 Burns AS, Lee BS, Ditunno Jr JF, Tessler A. Patient selection for clinical trials: the reliability of the early spinal cord injury examination. $J$ Neurotrauma 2003; 20: $477-482$.

14 Steeves J, Fawcett J, Tuszynski M. Report of international clinical trials workshop on spinal cord injury February 20-21, 2004, Vancouver, Canada. Spinal Cord 2004; 42: 591-597.

15 Kleitman N. Keeping promises: translating basic research into new spinal cord injury therapies. J Spinal Cord Med 2004; 27: 311-318.

16 Curt A, Schwab ME, Dietz V. Providing the clinical basis for new interventional therapies: refined diagnosis and assessment of recovery after spinal cord injury. Spinal Cord 2004; 42: 1-6.

\section{Websites relevant to clinical trials:}

http://www.consort-statement.org/statement/ revisedstatement.htm

http://ohrp.osophs.dhhs.gov/irb/irb_appendices.htm http://ohrp.osophs.dhhs.gov/irb/irb_guidebook.htm 